\title{
CONDITIONED MEDIUM INCREASES THE RATE OF NEURITE ELONGATION: SEPARATION OF THIS ACTIVITY FROM THE SUBSTRATUM-BOUND INDUCER OF NEURITE OUTGROWTH ${ }^{1}$
}

\author{
FRANK COLLINS ${ }^{2}$ AND ANDREA DAWSON \\ Department of Anatomy, University of Utah College of Medicine, Salt Lake City, Utah 84112
}

Received December 28, 1981; Revised April 12, 1982; Accepted April 12, 1982

\begin{abstract}
Heart cell-conditioned medium supports extensive neurite outgrowth from dissociated parasympathetic neurons of the chicken embryo ciliary ganglion. We have shown previously that neurite outgrowth in this system depends on the deposition of a substratum-conditioning factor from conditioned medium onto the polyornithine culture substratum. However, in the presence of only the substratum-bound material, neurite outgrowth is never as extensive as in whole conditioned medium. The present report demonstrates that a different and soluble component of conditioned medium is required to achieve the rates of neurite elongation normally observed in whole conditioned medium. This second component, while unable by itself to support neurite outgrowth, is able to increase the rate of neurite elongation approximately 3 -fold within 30 to $60 \mathrm{~min}$ of its addition. This conclusion is based on direct time-lapse observations of the rate of elongation of individual neurites before and after the addition of fractions of conditioned medium previously depleted of the substratum-conditioning factor. Correlated with the effect of such fractions of conditioned medium on the rate of neurite elongation is a change in the morphology of the growth cones, which become larger and more spread. The activity of the soluble, elongation-promoting component of conditioned medium is nondialyzable and is sensitive to treatments known to affect proteins, such as repeated freeze-thawing, heating, and trypsinization. Fractions of conditioned medium which contain the elongation-promoting activity also contain all of the survival factor for parasympathetic neurons previously shown to be present in heart cell-conditioned medium. The methods described here represent a convenient new assay which we have used recently to demonstrate elongation-promoting factors with neuronal specificity in extracts of rat hippocampus.
\end{abstract}

Growth conditioned medium has proved to be a promising source material for characterizing a range of neurotrophic influences and isolating the responsible molecules. A large proportion of this work has been done using medium conditioned by embryonic heart cells in culture. Two distinct effects of heart cell-conditioned medium on nerve cells in culture which are relevant to the present discussion have been described recently: (1) the induction of neurite outgrowth and (2) prolonged neuronal survival.

(1) Conditioned medium has been shown to contain a substratum-conditioning factor which is a powerful inducer of neurite outgrowth when bound to the polyornithine substratum on which nerve cells are cultured (Collins, 1978b; Collins and Garrett, 1980). Within $20 \mathrm{~min}$ of

\footnotetext{
' We wish to thank Mark R. Lee for his excellent technical assistance This work was supported by Grant NS15130 from the National Institutes of Health.

${ }^{2}$ To whom correspondence should be addressed.
}

the addition of conditioned medium to dissociated parasympathetic ciliary ganglion neurons, the majority of neurons have formed growth cones, and by $60 \mathrm{~min}$, greater than $80 \%$ of the neurons have extended at least 1 (and typically 3 to 5) actively elongating neurites (Collins, 1978a). The active factor must attach to the culture substratum in order to induce neurite outgrowth (Collins, 1978b; Collins and Garrett, 1980). Moreover, when the factor is painted onto the substratum in a narrow pathway, neurite outgrowth is channeled precisely along this pathway (Collins and Garrett, 1980). Not only do embryonic heart cells release this substratum-conditioning factor into their culture medium but they also deposit an identical biological activity into the extracellular matrix microexudate which they deposit directly onto their culture substratum (Collins, 1980). This substratum-conditioning factor may be closely related to those components of the in vivo extracellular matrix which support and direct neurite outgrowth.

(2) Dissociated parasympathetic ciliary ganglion neu- 
rons on a polyornithine substratum do not survive for more than $24 \mathrm{hr}$ in typical culture media supplemented only with animal sera. However, in heart or muscle cellconditioned medium, survival times of up to several weeks have been reported (Helfand et al,, 1976; Nishi and Berg, 1979). During this period in culture, some of the normal events of neuronal maturation, such as the increase in size of the neuronal soma, occur on schedule (Varon et al., 1979; Nishi and Berg, 1979). The survivalpromoting activity in heart cell-conditioned medium is different from the substratum-conditioning factor. The two activities can be easily separated from each other by taking advantage of the fact that the substratum-conditioning factor attaches to the polyornithine substratum, whereas the survival factor does not (Collins, 1978b; Adler and Varon, 1980).

The present paper reports an additional effect of heart cell-conditioned medium on neurite outgrowth. It became apparent in our work that fractions of conditioned medium which contained the substratum-conditioning factor, while they supported active neurite outgrowth, were incapable, by themselves, of reproducing the high rates of neurite elongation and the spread growth cones which are characteristic of the effects of the whole conditioned medium. Therefore, we have examined other fractions of conditioned medium for their ability to restore these features of neurite outgrowth. As reported here, conditioned medium contains a component (different from the substratum-conditioning factor) which increases the rate of neurite elongation approximately 3fold within 30 to 60 min of its addition.

\section{Materials and Methods}

Nerve cells. Ciliary ganglia were removed from stage 33 white Leghorn chicken embryos and dissociated into single cells using trypsin as previously described (Collins, 1978a). Before use, dissociated ganglia were placed in plastic tissue culture dishes in culture medium, F12FCS10 (see below), for $3 \mathrm{hr}$ in order to remove the non-neuronal cells (almost all of which adhered to the culture dishes) from the neurons (which did not adhere). The suspension of single, dissociated neurons then was plated onto a dish containing a "conditioned" substratum (see below) for subsequent observation of neurite elongation.

Culture conditions. Culture medium (F12FCS10) consisted of Ham's F12 medium (Gibco) containing $10 \%$ fetal calf serum (Irvine Scientific). "Conditioned" substrata for supporting neurite outgrowth were prepared as follows: plastic tissue culture dishes (Falcon) were coated with polyornithine as previously described (Collins, 1978a). The polyornithine-coated dishes then were incubated with heart cell-conditioned medium (see below) for 16 to $18 \mathrm{hr}$ at $4^{\circ} \mathrm{C}$ in order to coat the dish with a substratum-conditioning factor which induces neurite outgrowth from ciliary ganglion neurons (Collins, 1978b). An increase in the amount of the substratum-bound inducer will, by itself, increase somewhat the average length of extended neurites (Collins, 1978a). Therefore, in order to maximize the effect of the soluble conditioned medium fractions on neurite outgrowth, we have treated the substratum with just sufficient substratum-condi- tioning factor (determined by prior assay) to insure the initiation of outgrowth.

Heart cell-conditioned medium. A confluent monolayer of heart cells, prepared by tryptic dissociation of embryonic hearts from stage 33 chicken embryos, was cultured for $72 \mathrm{hr}$ in F12FCS10. The conditioned medium was removed from the monolayer and centrifuged at $10,000 \times g$ for $30 \mathrm{~min}$ to remove any cell debris (Collins, 1978a).

Fractionation of conditioned medium. The substratum-conditioning factor was removed from conditioned medium by either one of two procedures: (1) $15 \mathrm{ml}$ of heart cell-conditioned medium was placed successively for $8 \mathrm{hr}$ each in three $100 \times 15 \mathrm{~mm}$ polyornithine-coated plastic tissue culture dishes. The substratum-conditioning factor becomes bound to the polyornithine during this procedure so that the resultant polyornithine-passaged fraction has been depleted of this factor (Collins, $1978 \mathrm{~b})$. (2) Heart cell-conditioned medium was passed under nitrogen pressure through a filtration membrane (Amicon) with a 100,000 -dalton cutoff. The substratumconditioning factor is retained by the membrane so that the resultant ultrafiltrate fraction has been depleted of this factor ( $F$. Collins, unpublished observation).

Measurement of neurite lengths. At the times indicated in the text, cultures were fixed in $2 \%$ glutaraldehyde. The total length of all neurites present on each neuron was measured using a calibrated eyepiece reticle. These data were used to calculate the mean total length of neurites per neuron for each culture. The calculations were based on data only from neurons which had at least one neurite greater than $15 \mu \mathrm{m}$ in length (i.e., about one neuronal soma diameter).

Measurement of elongation rate. Dissociated ciliary ganglion neurons were plated onto a conditioned substratum in F12FCS10. The cultures were gassed in an atmosphere of $5 \% \mathrm{CO}_{2}, 95 \%$ air for several minutes and the lids were sealed against evaporation and $\mathrm{pH}$ change with silicone vacuum grease. The dishes were fastened to the stage of an inverted microscope equipped with phase contrast optics and maintained at $37^{\circ} \mathrm{C}$ with an air curtain incubator. A single microscopic field (usually containing 3 to 5 neurons) was followed by continuous recording of the field on videotape using television timelapse equipment. After $120 \mathrm{~min}$, the medium was changed either to fresh F12FCS10 or to either the ultrafiltrate or polyornithine-passaged fractions of conditioned medium and recorded for an additional 2 to $6 \mathrm{hr}$. The length of individual neurites (from the point of exit from the cell body to the center of the growth cone) was measured directly from the monitor screen through which the videotapes were playcd back. Since neurites adhere firmly to the substratum along their entire length, the increase in their length accurately reflects the distance traversed by their growth cones during the successive 30 min intervals for which rates of elongation were measured. This was established by careful examination of the continuous recordings of growth cone movement on videotape. If a neurite happened to branch during the period of observation, both new branches usually elongated at the same rate. The branch that appeared lower in the microscopic field was chosen arbitrarily for sub- 
sequent measurements. The rate of elongation of most neurites reached a constant value within $60 \mathrm{~min}$ after plating (i.e., 60 min before the medium was changed). Neurites which changed their rate of elongation by more than $\pm 50 \%$ during the $60 \mathrm{~min}$ immediately preceding the medium change ( $28 \%$ of the total) are not considered in the results.

Neuronal survival. The activity of the survival-promoting factor in heart cell-conditioned medium was assayed by the morphological integrity of the neuronal soma (Varon et al., 1979). The distinction between the intact, phase-refractile somas and the lysed, phase-dark somas was easily made. In unconditioned medium on a conditioned substratum, more than $95 \%$ of the ciliary ganglion neuronal somas are lysed within 18 to $24 \mathrm{hr}$ in the absence of any survival-promoting agents. In the presence of conditioned medium, greater than $80 \%$ of the neuronal somas are intact after $36 \mathrm{hr}$, which is the period of our assay for survival.

Enzymatic and other treatments. In order to test the susceptibility of the elongation rate-promoting activity to proteolytic enzymes, serum proteins were eliminated by producing serum-free conditioned medium. This was Inade as described above for heart cell-conditioned medium except that the heart cell monolayer was incubated for $48 \mathrm{hr}$ in Ham's F12 medium without added serum. The polyornithine-passaged fraction of this serum-free conditioned medium was subjected to the treatments indicated under "Results" and then placed on ciliary ganglion neurons in order to determine if the ability of the conditioned medium to enhance the rate of neurite elongation had been affected. Exposure to trypsin was accomplished by adding the equivalent of $50 \mu \mathrm{g} / \mathrm{ml}$ of crystalline trypsin bound to agarose beads (Sigma) to the conditioned medium for $30 \mathrm{~min}$ at $37^{\circ} \mathrm{C}$. The trypsin then was removed by pelleting the beads so that the medium could be assayed for elongation rate-promoting activity. Incubation at $37^{\circ} \mathrm{C}$ for $30 \mathrm{~min}$ in the absence of trypsin had no significant effect on the activity (Table IV).

\section{Results}

Neurites extended in the presence of only the substratum-bound material from conditioned medium are typically much shorter than those extended after a comparable period of outgrowth in whole conditioned medium (Table I, compare rows 1 and 2). In this experiment, the mean neurite length was approximately 4-fold greater in whole conditioned medium than it was in the presence of only the substratum-bound material from this conditioned medium.

This suggests that components of conditioned medium other than those which bind to the polyornithine substratum are required to obtain the full effect of conditioned medium on neurite outgrowth. To test this possibility, fractions of conditioned medium were prepared from which the substratum-bound inducer of neurite outgrowth had been removed. Two different methods were used to prepare such fractions (see "Materials and Methods"). One method resulted in an ultrafiltrate fraction; the other produced a polyornithine-passaged fraction. Both of these fractions were judged to be free of the

\section{TABLE I}

The effect of whole conditioned medium and of the ultrafiltrate subfraction on neurite lengths

Culture substrata were polyornithine-coated dishes used as such or after "conditioning" by preincubation with heart cell-conditioned medium. Dissocialed ciliary ganglion neurons were plated on the appropriate dishes either in (1) heart cell-conditioned medium; (2) unconditioned medium, F12FCS10; or (3) the ultrafiltrate fraction of heart cell-conditioned medium. After $2 \% / 4 \mathrm{hr}$, the cultures were fixed in $2 \%$ glutaraldehyde and the total neurite length per initiated neuron was measured for at least 50 neurons per culture. Given are the mean \pm SD for each culture.

\begin{tabular}{llcc}
\hline Substratum & Medium & $\begin{array}{c}\text { No. of } \\
\text { Neurons }\end{array}$ & $\begin{array}{c}\text { Mean Total } \\
\text { Neurite } \\
\text { Length/Neuron }\end{array}$ \\
\hline $\begin{array}{c}\text { Polyornithine } \\
\begin{array}{c}\text { Conditioned } \\
\text { polyornithine }\end{array}\end{array}$ & Unconditioned & 57 & $408 \pm 94$ \\
$\begin{array}{c}\text { Conditioned } \\
\text { polyornithine }\end{array}$ & Ultrafiltrate & 52 & $471 \pm 81$ \\
\hline
\end{tabular}

substratum-bound inducer of neurite outgrowth by assays described below. These fractions were added to neurons growing on the substratum-bound material to determine if they were capable of restoring the neurite lengths observed in whole conditioned medium. Table I, row 3 , indicates that the addition of the ultrafiltrate fraction resulted in mean neurite lengths which were comparable to those obtained in whole conditioned medium. A similar result was obtained using the polyornithine-passaged fraction of conditioned medium (Fig. 1).

Effect on the rate of neurite elongation. The approximately 4-fold differences in neurite lengths reported above were manifested in a relatively brief 3 -hr period. This rapid effect suggests that the ultrafiltrate and polyornithine-passaged fractions of conditioned medium may affect the rate of neurite elongation directly. To test this, the rate of elongation of individual neurites was compared before and after the addition of one of the fractions. Table II presents the combined results of a number of these individual comparisons. The rate of elongation during the first $30 \mathrm{~min}$ after the addition of either of the conditioned medium fractions was not significantly different from the rate before their addition (Table II, column 4). However, between 30 and $60 \mathrm{~min}$, there was an approximately 3 -fold increase in the rate of elongation of individual neurites (Table II, column 5). The statistical significance of this effect was extremely high (Table II, column 5). This increased rate of elongation was maintained until observation of the cultures was discontinued, from 2 to $6 \mathrm{hr}$ after the addition of the conditioned medium fraction. In control cultures, to which only fresh unconditioned medium had been added, the average rate of elongation of individual neurites did not change significantly over the same time period (Table II).

Since neurites tend to branch after reaching a certain length, there are typically more elongating branches per neuron in the faster growing conditioned medium-treated cultures than in control cultures (Fig. 1). The greater number of elongating branches per neuron and the 3 -fold increase in their average rate of elongation are sufficient 

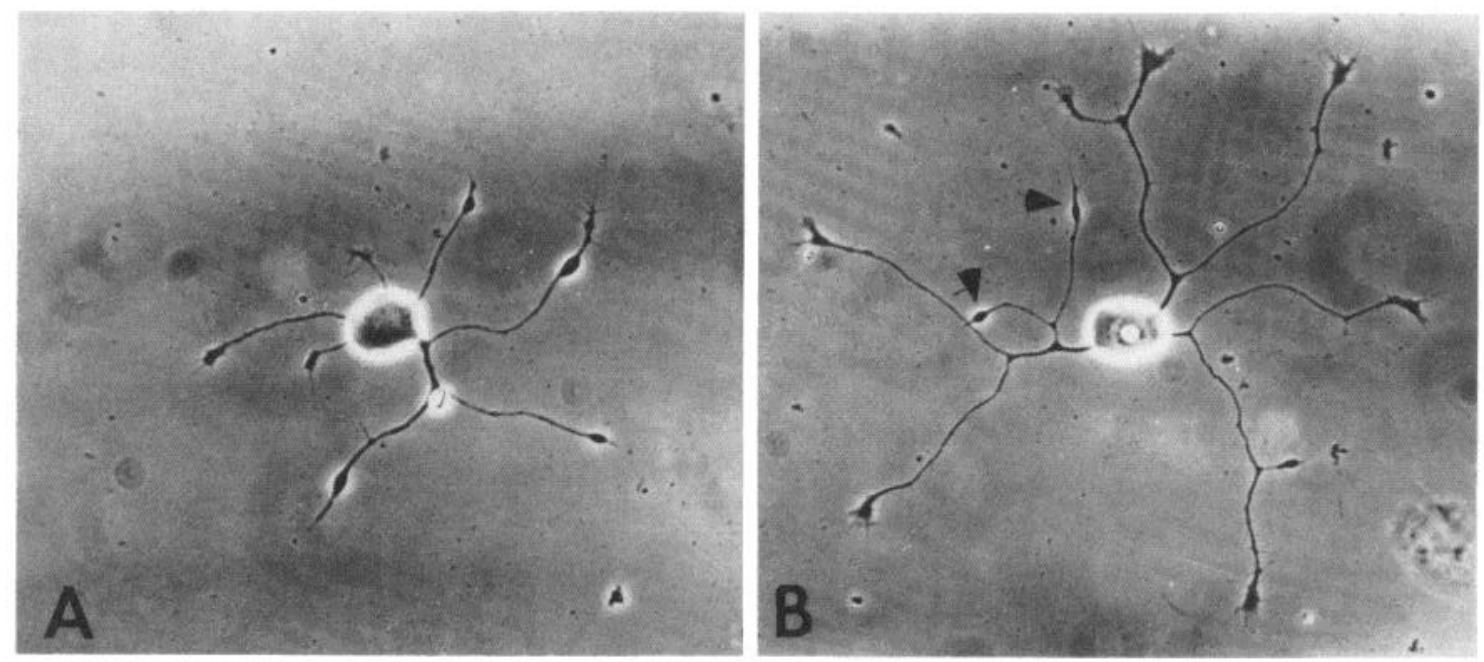

Figure 1. The effect on neuronal morphology of the polyornithine-passaged fraction of conditioned medium. Dissociated neurons were plated onto conditioned substrata in either unconditioned medium $(A)$ or in the polyornithine-passaged fraction of conditioned medium $(B)$. Note both the greater neurite lengths and the more spread growth cones in the treated culture $(B)$. The arrowheads in $B$ indicate unspread growth cones which are more characteristic of the untreated control neurons in $A$. Magnification $\times 530$.

TABLE II

The effect of conditioned medium fractions on the rate of neurite elongation

Polyornithine-coated dishes were conditioned by incubation with heart cell-conditioned medium. Dissociated ciliary ganglion neurons were plated onto these dishes in unconditioned medium (F12FCS10). Time-lapse video recording was begun and the cultures were followed for 120 min, at which time the medium was changed to either (1) fresh, unconditioned medium (control cultures) or (2) the ultrafiltrate or polyornithinesoluble fractions of conditioned medium (experimental cultures). The results of several experiments using both conditioned medium fractions have been combined. The significance levels ( $p$ values) were calculated from paired $t$ tests which compared the elongation rate of individual neurites before the medium change to their elongation rate 0 to 30 or 30 to $60 \mathrm{~min}$ after the medium change.

\begin{tabular}{lcccc}
\hline \multirow{2}{*}{ Culture } & & \multicolumn{3}{c}{ Mean Elongation Rate } \\
\cline { 3 - 5 } & No. of Neurites & $\begin{array}{c}\text { 0-30 Min Before } \\
\text { Medium Change }\end{array}$ & $\begin{array}{c}0-30 \text { Min After } \\
\text { Medium Change }\end{array}$ & $\begin{array}{c}\text { 30-60 Min After } \\
\text { Medium Change }\end{array}$ \\
\hline Control & 14 & & $26(p>0.2)$ & $22(p>0.2)$ \\
Experimental & 27 & 22 & $37(p=0.15)$ & $68(p<0.001)$ \\
\hline
\end{tabular}

to account for the increase in total neurite length per neuron in the treated cultures (Table I). One additional effect of conditioned medium which might have led to increased neurite lengths does not seem to occur: the conditioned medium fractions did not cause neurite outgrowth to begin sooner than in control cultures (data not shown).

Effects on growth cone morphology. In addition to their dramatic effects on the rate of neurite elongation, the ultrafiltrate and polyornithine-passaged fractions of conditioned medium have a definite effect on the morphology of the growth cones. The growth cones of control neurons (i.e., those cultured on the substratum-bound material in unconditioned medium) tend to be relatively small and not flattened onto the substratum; often there is a refractile bulge just behind the growth cone (Fig. $1 A$ ). In contrast, in cultures which were exposed to either the ultrafiltrate or polyornithine-passaged fractions of conditioned medium, the majority of growth cones are large, well spread, and free of bulges on the terminal portion of the neurite (Fig. $1 B$ ). Our time-lapse observations show that the less spread growth cones characteristic of the control cultures are capable of moving over the substratum, although their average rate of translo- cation is lower than the more spread growth cones in treated cultures. The difference in growth cone morphology between treated and control cultures is only a quantitative difference, since either culture contains a few growth cones characteristic of the other (indicated by the arrowheads in Fig. 1). Thus, the conditioned medium fractions allow neurons to maintain a greater proportion of their growth cones in a well spread condition.

Relationship to other activities of conditioned medium. The agent responsible for enhancing the rate of neurite outgrowth in the two conditioned medium fractions used above is clearly different from the substratumconditioning factor. To be certain that the substratumbound inducer had been removed from these fractions, each batch of depleted conditioned medium was assayed for substratum-bound inducer in the following ways: (1) the fraction was added directly to dissociated ciliary ganglion neurons on a polyornithine substratum (Table III) and (2) the fraction was incubated overnight in polyornithine-coated dishes; the dishes then were washed and dissociated ciliary ganglion neurons were plated on in unconditioned medium (Collins, 1978b). Whole conditioned medium of the same batch was run as a control in both of the above procedures. Since initiation of neu- 
rite outgrowth depends upon the presence of the substratum-conditioning factor (Collins, 1978b), the extent of removal of this factor was determined by measuring the maximum percentage of the neurons which were able to extend neurites. The results (Table III) indicated that, within the limits of the sensitivity of the assay, there was no remaining substratum-bound inducer. In other words, the fractions of conditioned medium were unable by themselves to promote the initiation of neurite outgrowth, although they were able to increase the rate of neurite elongation and affect the morphology of growth cones as described above.

Heart cell-conditioned medium also is able to prolong the survival of ciliary ganglion neurons in culture (Helfand et al., 1976; Nishi and Berg, 1979). This survivalpromoting activity is readily separated from the substratum-bound inducer (Collins, 1978b; Adler and Varon, 1980). To determine if the survival-promoting activity is present in those fractions of conditioned medium which increase the rate of neurite elongation, the survival of dissociated ciliary ganglion neurons cultured in those fractions was compared to the survival in whole conditioned medium and in unconditioned medium. The results (Table III) clearly indicate that the survival-promoting activity is retained in those fractions of conditioned medium which also increase the rate of neurite elongation.

Chemical characteristics. As a preliminary step in determining the chemical nature of the elongation-promoting activity, the polyornithine-passaged fraction of serum-free conditioned medium was subjected to various treatments and then assayed for its ability to increase the average neurite length of dissociated ciliary ganglion neurons cultured for $2 \mathrm{hr}$ in its presence. To the extent that the treatments had destroyed the elongation-promoting material, one would expect the neurite lengths observed to approach those seen in control, unsupplemented cultures. The results (Table IV) indicate that the elongation-promoting activity is nondialyzable, heat sensitive, destroyed by repeated freeze-thawing, and sensi-

TABLE III

Assay of conditioned medium and subfractions for neurite-initiating and survival-promoting activities

Polyornithine-coated dishes were conditioned by incubation with heart cell-conditioned medium. Dissociated ciliary ganglion neurons were plated onto these dishes in one of the media indicated above. The percentage of 200 neurons per culture which were able to initiate neurite outgrowth was measured at intervals up to $12 \mathrm{hr}$. The maximum recorded percentage is given. The maximum occurred at $2 \mathrm{hr}$ in whole conditioned medium. For assay of neuronal survival, the conditions were as above except that the whole conditioned medium and subfractions were diluted serially 2 -fold with unconditioned medium before use. The titer was calculated as the greatest dilution which still gave $50 \%$ or greater neuronal survival at $36 \mathrm{hr}$.

\begin{tabular}{lcc}
\hline \multicolumn{1}{c}{ Medium } & $\begin{array}{c}\text { Maximum Percentage } \\
\text { of Initiated Neurons }\end{array}$ & $\begin{array}{c}\text { Titer of Survival- } \\
\text { promoting Activity }\end{array}$ \\
\hline Conditioned medium & $\%$ & \\
Polyornithine-soluble & 86 & $1: 16$ \\
$\quad$ subfraction & 0 & $1: 16$ \\
Ultrafiltrate subfraction & 0 & $1: 16$ \\
Unconditioned medium & 0 & 0 \\
\hline
\end{tabular}

\section{TABLE IV}

The effect of various treatments on the elongation-promoting activity

Polyornithine-coated dishes were conditioned by incubation with heart cell-conditioned medium. Dissociated ciliary ganglion neurons were plated onto these dishes in unconditioned medium (F12FCS10). After 45 min, the medium was changed to the polyornithine-soluble fraction of serum-free heart cell-conditioned medium pretreated as indicated. Two hours after the medium change, cultures were fixed in $2 \%$ glutaraldehyde and the total neurite length per initiated neuron was measured on 25 neurons per culture. The results of two independent experiments are given here. The following procedure was used to normalize the values for combined presentation: the mean total neurite length per initiated neuron in a control culture (in unconditioned medium, F12FCS10) was subtracted from the analogous value in each experimental culture. The resultant values gave the increase in length due to the added fractions. These values then werc divided by the value for the culture in which there was no pretreatment of the polyornithinesoluble fraction to give the percentage of activity remaining after each treatment. 'The values given are the average percentage \pm the range for two experiments.

\begin{tabular}{lc}
\hline \multicolumn{1}{c}{ Treatment } & Percent Remaining Activity \\
\hline None & 100 \\
Dialysis (retentate) & $82 \pm 13$ \\
$37^{\circ} \mathrm{C}$ for 30 min & $91 \pm 16$ \\
$50 \mu \mathrm{g} / \mathrm{ml}$ of bound trypsin & $-6 \pm 12$ \\
Five freeze-thawings & $18 \pm 17$ \\
$80^{\circ} \mathrm{C}$ for 30 min & $-2 \pm 19$ \\
\hline
\end{tabular}

tive to trypsin. This suggests that a protein component is essential for the activity.

\section{Discussion}

Heart cell-conditioned medium supports rapid and extensive neurite outgrowth from dissociated neurons of the parasympathetic ciliary ganglion in vitro (Helfand et al., 1976; Collins, 1978a). The results that we have reported here indicate that at least two distinct components of conditioned medium are required for this effect. One is a factor which binds to the culture substratum and is required for neurite outgrowth to occur, while the second is a soluble component which is unable by itself to support neurite outgrowth but which increases the rate of neurite elongation approximately 3 -fold within 30 to $60 \mathrm{~min}$ of its addition. Together these two components reconstitute the effects of conditioned medium on neurite outgrowth.

We have shown in a series of papers that the induction of neurite outgrowth by heart cell-conditioned medium requires the deposition of a substratum-conditioning factor onto the polyornithine substratum (Collins, 1978b, 1980; Collins and Garrett, 1980). In the absence of this substratum-bound material, neurite outgrowth fails to occur in this culture system. If a pathway of the substratum-conditioning factor is painted onto the culture dish, elongating neurites will change their direction of movement in order to remain in contact with that portion of the substratum to which the substratum-conditioning factor is attached (Collins and Garrett, 1980).

Thus, the substratum-bound material from conditioned medium provides an adequate and preferred substratum for neurite elongation. However, as reported here, in the absence of those components of conditioned 
medium which do not bind to the polyornithine substratum (i.e., when neurons are cultured on the substratumbound material in unconditioned culture medium), rates of neurite elongation are noticeably less and growth cones are smaller and less well spread than they are when the neurons are cultured in whole conditioned medium. By removing the substratum-conditioning factor, we have produced fractions of conditioned medium which are unable by themselves to support neurite outgrowth but, when added to neurons growing on the substratumbound material, are able to restore the rates of elongation and spread growth cones characteristic of whole conditioned medium. The effects of these fractions on the elongation rate and growth cone morphology may be related. Since motility of the growth cone leads to elongation, it is possible that the change in the morphology of the growth cone reflects a change in its functional activity, induced by conditioned medium, which results in the increased rate of elongation.

The agent responsible for these effects is trypsin sensitive, nondialyzable, heat labile, and susceptible to repeated freeze-thawing. 'Ihis suggests that the agent is a protein. This agent is readily separated from the substratum-conditioning factor in either one of two ways: (1) by pressure dialysis through a membrane with a nominal 100,000-dalton cutoff, in which case the substratum-conditioning factor is retained, while the elongation-promoting factor is in the filtrate or (2) by passage over a number of polyornithine-coated dishes, which removes the substratum-conditioning factor, leaving the elongation-promoting factor in solution. Both the ultrafiltrate and the polyornithine-passaged fractions contain not only the elongation-promoting activity but they also contain all of the detectable survival-promoting activity for parasympathetic neurons present in the original conditioned medium. An interesting question for future research will be to determine if these two activities are effects of the same component.

The methods that we have used here represent a convenient new assay for potential neurotrophic factors.
On a polyornithine substratum pretreated with the appropriate amount of substratum-conditioning factor, neurite outgrowth is poised to respond to agents which are capable of increasing the rate of neurite elongation. Recently, we have used the assay described in this paper to demonstrate that rat hippocampal extracts contain two elongation-promoting factors which differ in their neuronal specificity (Crutcher and Collins, 1982). These hippocampal agents, one of which is antigenically related to nerve growth factor, may be involved in the sprouting responses induced in vivo by hippocampal deafferentation.

\section{References}

Adler, R., and S. Varon (1980) Cholinergic neuronotrophic factors. V. Segregation of survival and neurite promoting activities in heart-conditioned medium. Brain Res. 118: 437448 .

Collins, F. (1978a) Axon initiation by ciliary neurons in culture. Dev. Biol. 65: 50-57.

Collins, F. (1978b) Induction of neurite outgrowth by a conditioned medium factor bound to the culture substratum. Proc. Natl. Acad. Sci. U. S. A. 75: 5210-5213.

Collins, F. (1980) Neurite outgrowth induced by the substrate associated material from nonneuronal cells. Dev. Biol. 79: 247-252.

Collins, F., and J. E. Garrett, Jr. (1980) Elongating nerve fibers are guided by a pathway of material released from embryonic nonneuronal cells. Proc. Natl. Acad. Sci. U. S. A. 77: 62266228.

Crutcher, K. A., and F. Collins (1982) In vitro evidence for two distinct hippocampal growth factors: Basis of neuronal plasticity? Science 217: 67-68.

Helfand, S. L., G. A. Smith, and N. K. Wessells (1976) Survival and development in culture of dissociated parasympathetic neurons from ciliary ganglia. Dev. Biol. 50: 541-547.

Nishi, R., and D. K. Berg (1979) Survival and development of ciliary ganglia neurons grown alone in cell culture. Nature 277: 232-234.

Varon, S., M. Manthorpe, and R. Adler (1979) Cholinergic neuronotrophic factors. I. Survival, neurite outgrowth, and CAT activity in monolayer cultures from chick embryo ciliary ganglia. Brain Res. 173: 29-45. 